\title{
Aberration-Corrected HAADF-STEM Studies of Nano-Gold/Titania Catalysts
}

\author{
K. Yoshida, ${ }^{*}, *$ J.C. Hernandez-Garrido, ${ }^{* * *}$ J.M. Thomas, ${ }^{* * *}$ E.D. Boyes, ${ }^{*}$ P.A. Midgley, ${ }^{* * *}$ and \\ P.L. Gai*
}

* The Nanocentre, Department of Chemistry, Department of Physics and Department of Electronics, The University of York, York Y010 5DD, U.K.

** Nanostructures Research Laboratory, Japan Fine Ceramics Center, Nagoya, 456-8587, Japan

*** Department of Materials Science and Metallurgy, University of Cambridge, Pembroke Street, Cambridge, CB2 3QZ, U.K.

Gold exhibits remarkably high catalytic activity at the nanoscale when finely dispersed on selected metal oxide supports [1]. Gold-titania catalysts are very attractive systems for a number of reactions in heterogeneous catalysis. They are utilized for low-temperature carbon monoxide oxidation and the direct epoxidation of propene to propene oxide [2a,b]. Over the past years considerable efforts have been made by a number of research groups, primarily using chemical methods, to explore the catalytic performance of gold nanoparticles [3]. However the detailed nanostructure and the location of the nanoparticles in the $\mathrm{Au} / \mathrm{TiO}_{2}$ system at the atomic scale, which could be vital for catalytic activity, selectivity and the nanoparticle stability, are not well understood. Here we have investigated the fine structure of the catalysts directly, using a double aberration-corrected (AC) HAADF-STEM to obtain better insights into the atomic structure and location of the nanoparticles on the support. These are key steps in understanding and facilitating the development of improved catalysts.

Atomically dispersed and slightly larger nanoclusters of gold in a $\mathrm{Au} / \mathrm{TiO}_{2}$ selective oxidation catalyst were imaged in a double aberration-corrected JEOL 2200FS TEM/STEM at the University of York. Both AC-TEM and AC HAADF-STEM images were recorded. FIG.1 is an AC-TEM image of $\mathrm{Au} / \mathrm{TiO}_{2}$ catalyst. The lattice spacings indicate that the support is anatase form of titania. FIG.2(a) shows AC HAADF-STEM micrographs. The Z-contrast images indicate that the nanoparticles are localized at grain boundaries of titania grains. FIG.2(b) shows high resolution AC HAADF-STEM image of the sample indicating the different grain orientations at the atomic level. The lattice spacings of the support are consistent with the anatase form of titania. We have recorded individual gold atoms and sub-nanometer clusters of gold on the crystalline support and they are illustrated in FIG.3. The results indicate that the individual metal atoms and ultra small clusters of only a few atoms may be dominant in determining the activity and selectivity of the catalyst. The location of the nanoparticles at the grain boundaries, confirmed by tomography studies [4], seems likely to influence the stability significantly and hence continuing catalytic activity of the nanoparticles.

\section{References}

[1] M. Haruta, Catal. Today. 36 (1997) 153.

[2] (a) J. D. Grunwaldt et al., J.Phys.Chem.(b) 103 (1999) 1002. [b] T.A. Nijhuis et al., Catal. Today. 117 (2006) 84

[3] W. Deng et al. Appl. Catal.A. 291 (2005) 126.

[4] J. M. Thomas. et al., Angew. Chem. 48 (2009) 3904.

[5] KY thanks the JSPS for fellowships. JCHG and PAM acknowledge financial support from the European Union under the Framework 6 program for an Integrated Infrastructure Initiative, Ref:: 026019 ESTEEM. Claus H. Christensen is kindly acknowledged for providing the sample. The 
support of the University of York, Yorkshire Forward/ERDF(EU), and JEOL for the York-JEOL Nanocentre at the University of York is gratefully acknowledged.

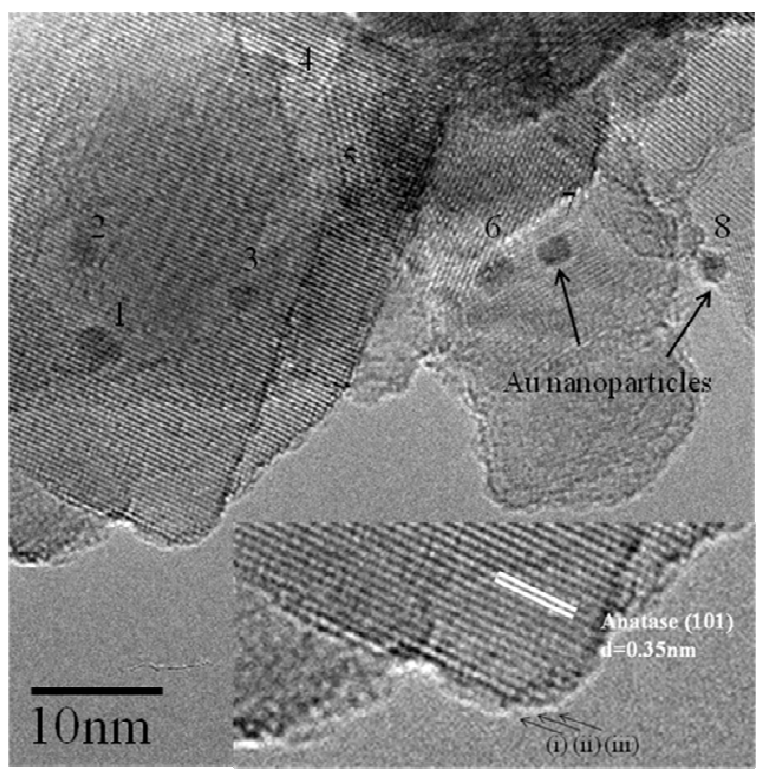

FIG. 1 Cs corrected HRTEM of $\mathrm{Au} / \mathrm{TiO}_{2}$ catalyst. Surface roughness of $1.05 \mathrm{~nm}$ is estimated by anatase (101) planes (i), (ii) and (iii).
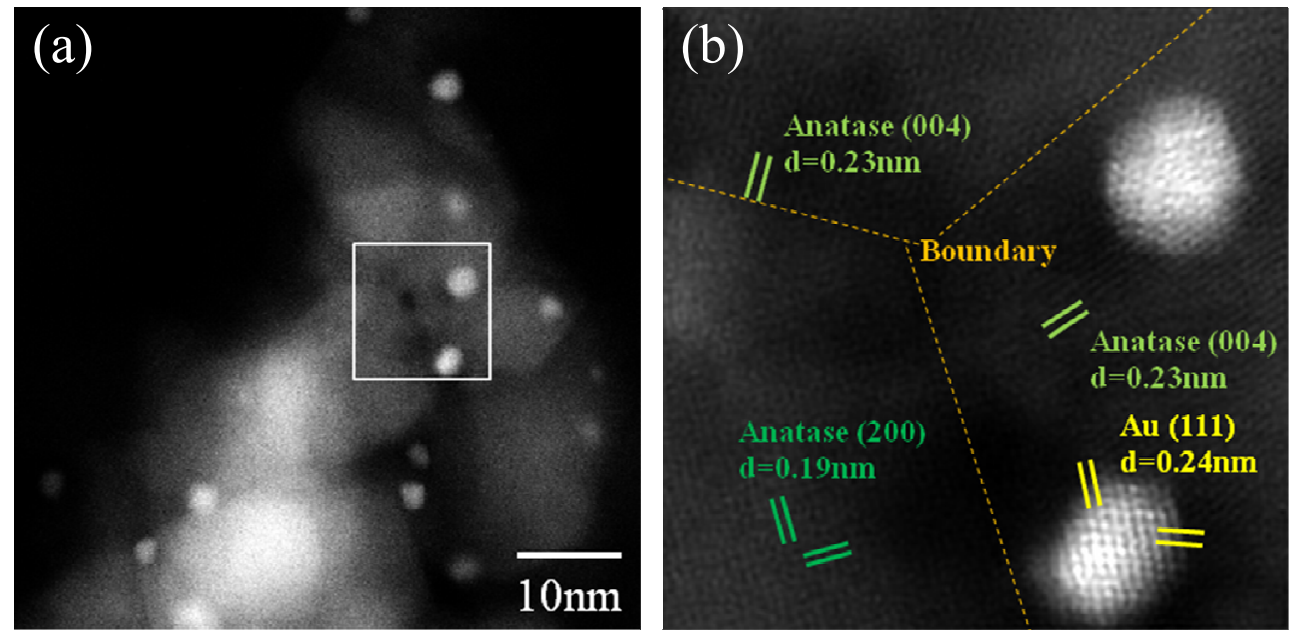

FIG. 2 (a)AC-HAADF-STEM: nanoparticles at grain boundaries (b) AC HAADF-STEM showing lattice resolution of titania and gold.
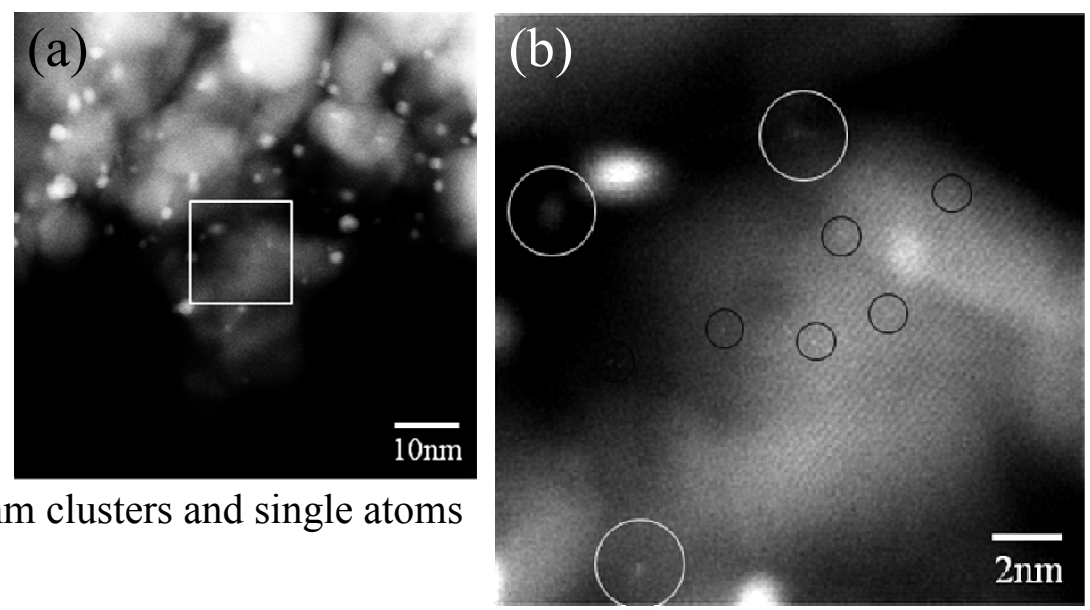

FIG. 3 Sub-nm clusters and single atoms of gold. 